\title{
ALTERACIONES DE LA SECRECIÓN NOCTURNA DE MELATONINA Y NEUROPATÍAS ÓPTICAS
}

\author{
ALTERATIONS IN NOCTURNAL MELATONIN LEVELS \\ IN PATIENTS WITH OPTIC NEUROPATHIES
}

\author{
PÉREZ-RICO C ${ }^{1}$, DE LA VILLA P ${ }^{1}$, BLANCO R ${ }^{1}$, GERMAIN $F^{1}$, PAZ-MORENO J ${ }^{2}$, \\ ARRIBAS-GÓMEZ I ${ }^{1}$
}

\begin{abstract}
RESUMEN
Objetivo: Evaluar la supresión de la secreción nocturna de melatonina inducida por exposición a la luz en pacientes con neuropatías ópticas bilaterales. Métodos: Estudio clínico de casos controles, observacional y prospectivo. Tamaño muestral de 20 pacientes distribuidos en 3 grupos: Grupo A ( $n=5$, Sujetos Sanos Controles), Grupo B ( $\mathrm{n}=10$, Pacientes Experimentales) y Grupo $\mathrm{C}(\mathrm{n}=5$, Sujetos Controles Ciegos). Se analiza la mejor agudeza visual corregida LogMAR, la desviación media en perimetría estática automatizada, el espesor medio de la capa de fibras nerviosas retinianas mediante Tomografía de Coherencia Óptica y los registros de electrorretinografía multifocal ( $m f E R G)$. Se realizan determinaciones de melatonina en saliva por radioinmunoensayo tras exposición a una luz de 600 lux durante 1 hora (Test de supresión nocturna de melatonina).
\end{abstract}

Resultados: Se encontraron diferencias estadísticamente significativas entre los grupos. No se observaron cambios en los registros de $m f$ ERG. El test de

\begin{abstract}
Objective: To study nocturnal melatonin suppression induced by exposure to light in patients with bilateral optic neuropathies.

Methods: Observational, prospective case control study. Twenty patients were included in this study and distributed in 3 groups: Group A ( $n=5$, Healthy Control Subjects), Group B ( $\mathrm{n}=10$, Experimental Patients) and Group C ( $\mathrm{n}=5$, Blind Control Subjects). LogMAR best-corrected visual acuity, standard automated perimetry mean deviation, retinal nerve fiber layer thickness by Optical Coherence Tomography and multifocal electroretinograpy ( $m f$ ERG) were used to evaluate the changes. Melatonin was analysed in the saliva by radioimmunoassay after exposure to light (600 lux for 1 hour) (nocturnal melatonin suppression test).

Results: Statistically significant differences between the groups were found. No changes in the $m f$ ERG results were detected. The nocturnal melatonin suppression test was positive in all cases in Group A, 50 \% in Group B and none in Group C.
\end{abstract}

\footnotetext{
Recibido: 30/5/07. Aceptado: 24/4/09.

Servicio de Oftalmología y Servicio de Análisis Clínicos. Hospital Universitario Príncipe de Asturias. Alcalá de Henares. Departamento de Fisiología y Cirugía. Facultad de Medicina. Universidad de Alcalá. España.

${ }^{1}$ Doctor en Medicina.

2 Licenciado en Medicina.

Correspondencia:

Consuelo Pérez Rico

Cl. Eduardo Mazón, n. ${ }^{2} \mathrm{C}$

28042 Madrid

España

E-mail: cintaperezrico@hotmail.com
} 
supresión nocturna de melatonina fue positivo en todos los casos del Grupo A, en el 50\% de los casos del Grupo B y en todos los casos del Grupo C fue negativo.

Conclusiones: El 50\% de los pacientes con neuropatías ópticas y pérdida visual severa exhiben alteraciones significativas en la secreción nocturna de melatonina, probablemente debido a una disfunción de las células ganglionares de la retina intrínsecamente fotosensibles (ipCGR).

Palabras clave: Melatonina, ritmos circadianos, núcleo supraquiasmático, efectos de la luz, neuropatías ópticas.
Conclusions: Half of the patients with optic neuropathies and severe visual loss were shown to suffer significant melatonin regulation anomalies, probably due to the dysfunction of the intrinsically photosensitive retinal ganglion cells (ipRGC) (Arch Soc Esp Oftalmol 2009; 84: 251-258).

Key words: Melatonin, circadian rhythms, suprachiasmatic nuclei, light effects, optic neuropathies.

\section{INTRODUCCIÓN}

La melatonina es una hormona sintetizada y segregada por la glándula pineal con una periodicidad circadiana (1). La luz fototransducida en las células ganglionares de la retina intrínsecamente fotosensibles (ipCGR) $(2,3)$, es conducida a través del tracto retinohipotalámico (TRH) al núcleo supraquiasmático del hipotálamo (NSQ), principal reloj circadiano de los mamíferos (4), que conecta la retina con la glándula pineal a través de una vía neural multisináptica (5).

La luz ejerce dos efectos sobre la secreción de melatonina (6): 1) los ciclos luz-oscuridad sincronizan el ritmo circadiano de su secreción, 2) una luz de suficiente intensidad y duración inhibe de forma brusca su secreción, de forma dosis-dependiente.

En el hombre, la melatonina plasmática es prácticamente de origen pineal, la hormona entra en el torrente circulatorio por difusión pasiva (7). Su secreción se incrementa pronto tras el inicio de la oscuridad, con un pico máximo en la mitad de la noche (entre las 2 y 4 a.m.), y gradualmente decae en la segunda mitad de la noche (7). Las concentraciones de melatonina en saliva son aproximadamente el $30 \%$ de los niveles plasmáticos, mostrando el mismo ritmo circadiano. Existe una perfecta correlación entre la melatonina pineal, plasmática, urinaria y salivar $(8,9)$.

En este trabajo, nos proponemos evaluar la integridad funcional del TRH y la respuesta neuroendocrina de la glándula pineal a la luz en pacientes con neuropatías ópticas severas bilaterales, mediante la determinación de melatonina en saliva por radioinmunoensayo (RIA). En este sentido, nos plantea- mos relacionar la supresión de la secreción nocturna de melatonina inducida por la exposición a una luz brillante de 600 lux durante 1 hora con la severidad de la pérdida de la función visual ocasionada por la lesión de las células ganglionares de la retina. La severidad de la neuropatía óptica será documentada y objetivada mediante técnicas diagnósticas morfológicas, como la tomografía de coherencia óptica (OCT) y funcionales, como la electrorretinografía multifocal ( $m f E R G)$.

\section{SUJETOS, MATERIAL Y MÉTODOS}

\section{Sujetos}

Se han estudiado 20 sujetos, en edades comprendidas entre 48 y 68 años, distribuidos en 3 grupos:

- Grupo A ( $\mathrm{n}=5)$ : Sujetos Sanos Controles, con criterios de exploración oftalmológica normal en ambos ojos (AO) y sin historia familiar de glaucoma.

Mejor agudeza visual corregida (MAVC) en escala $\log \mathrm{MAR}<+0,3$.

Presión intraocular $(\mathrm{PIO})<21 \mathrm{~mm} \mathrm{Hg}$.

Error refractivo $<5$ dioptrías de equivalente esférico o 3 dioptrías de astigmatismo.

Perimetría estática Humphrey (HVF) normal.

- Grupo B ( $\mathrm{n}=10)$ : Pacientes Experimentales reclutados en la Unidad de Neuro-oftalmología del Hospital Universitario Príncipe de Asturias, afectos de neuropatía óptica bilateral severa y con los siguientes criterios de inclusión en AO:

MAVC $\leq+1,00$.

Desviación media (DM) en HVF $<-12$ dB. 
Reducción del espesor medio de la capa de fibras nerviosas retinianas (CFNR) en $360^{\circ}$ inferior al percentil 1\% (Avg Thickness/OCT).

Función de la retina externa normal en $m f E R G$.

- Grupo C ( $n=5)$ : Sujetos Controles Ciegos, remitidos por la Organización Nacional de Ciegos Españoles (ONCE) y que cumplían los siguientes criterios en $\mathrm{AO}$ :

No percepción de luz (PL) consciente.

Reflejos pupilares a la luz abolidos.

Atrofia óptica diagnosticada oftalmoscópicamente.

Con excepción de historia de insomnio en algún paciente ciego, ninguno de los sujetos incluidos en el estudio presentaba enfermedad sistémica o psiquiátrica, ni se encontraba bajo tratamiento médico alguno. En ningún caso desempeñaban actividades laborales nocturnas. Todos los participantes firmaron el consentimiento informado de acuerdo a la Declaración Helsinki. El estudio fue aprobado por el Comité Ético de Hospital Universitario Príncipe de Asturias.

\section{Métodos}

Todos los sujetos controles y pacientes experimentales se sometieron a una exploración oftalmológica rutinaria: medida de la MAVC, refracción, reflejos pupilares, biomicroscopía con lámpara de hendidura, medida de PIO, estudio de fondo de ojo y retinografías. En los sujetos del Grupo A y B se realizaron una serie de exploraciones complementarias: HVF, examen OCT y pruebas $m f E R G$, para objetivar y documentar la severidad de la neuropatía óptica. Las medidas obtenidas en cada uno de los ojos fueron promediadas. En todos los casos se determinaron concentraciones de melatonina en saliva por RIA.

La medida de la MAVC se efectuó mediante un optotipo Logarithmic Visual Acuity (LogMAR) Chart 2000 «New ETDRS» de Precisión Visión. El estudio del campo visual se realizó mediante un perímetro computarizado Humphrey modelo 740 (Humphrey Instruments Inc.), utilizando una estrategia SITA 24-2 con un estímulo III de Goldmann. Se adaptó siempre la corrección apropiada para la distancia de examen y se incluyeron los datos correspondientes a una segunda exploración. Sólo se recogieron los resultados de los campos visuales con una fiabilidad aceptable (menos del $25 \%$ de pérdidas de fijación, falsos positivos y falsos negativos).

\section{Tomografía de coherencia óptica (OCT)}

Las exploraciones fueron realizadas mediante OCT Stratus TM 4.0.2 versión 0052 (Carl Zeiss Meditec, Inc, Dublín, California) y por un único explorador experimentado (JPM). Para el estudio del espesor de la CFNR peripapilares se utilizó el protocolo Fast RNFL Thickness 3,4. Este protocolo realiza 3 barridos circulares de 3,4 $\mathrm{mm}$ de diámetro en 1,92 segundos y los comprime en una única tomografía. Cada barrido contiene 256 A-scans. Todas las medidas fueron realizadas en las mismas condiciones de luminosidad ambiental y bajo dilatación pupilar, en todos los casos se utilizó fijación interna (luz diana verde en el interior del globo ocular). Se obtuvieron 3 tomografías con una señal adecuada (superior a 5) por cada ojo, que fueron archivadas para calcular valores promedio. En cada ojo, se estudió la medida del espesor medio de CFNR en 360: Avg Thickness, automáticamente calculada por el software de la OCT.

\section{Electrorretinografía multifocal ( $m f$ ERG)}

Para obtener los registros de $m f$ ERG se utilizó un Sistema de electrofisiología multifocal VERIS (EDI, San Mateo, USA). En todos los casos se dilataron las pupilas con colirio de tropicamida y de fenilefrina al 2,5\% y se colocó ante el ojo la corrección apropiada para la distancia de examen Los pacientes se adaptaron durante 15 minutos a la luminancia de la sala de exploración $\left(6 \mathrm{~cd} / \mathrm{m}^{2}\right)$ y el ojo contralateral fue ocluido. Se colocó un electrodo bipolar Burian-Allen tipo lente de contacto protegido con metilcelulosa sobre la córnea previamente anestesiada de forma tópica, y otro electrodo de masa en el lóbulo de la oreja derecha.

Se utilizó un estímulo constituido por 103 elementos hexagonales alineados en círculos concéntricos que subtienden un ángulo visual de $20-30^{\circ}$ a ambos lados del punto de fijación. Los estímulos fueron visualizados en un monitor de TV SONY Trinitron MultiSCAN modelo E500, con una frecuencia de $75 \mathrm{~Hz}$ y una luminancia máxima de 100$200 \mathrm{~cd} / \mathrm{m}^{2}$ (blancos) y mínima $<1 \mathrm{~cd} / \mathrm{m}^{2}$ (negros), siguiendo una secuencia-m-binaria pseudorandomizada. El contraste entre los estímulos blancos y negros fue del $90 \%$ o mayor y en todo momento se monitorizó la fijación del paciente. Las señales se amplificaron por 100.000 y se estableció un filtraje 
con un rango entre 10 y $300 \mathrm{~Hz}$. La duración total del registro fue de 8 minutos. Los registros fueron analizados mediante un software propio desarrollado en MatLab.

\section{Determinaciones de melatonina en saliva}

Las concentraciones de melatonina en saliva fueron determinadas por RIA (Melatonin direct RIA saliva, RE29371, IBL Immuno Biological Laboratorios, Germany). Este test presenta una sensibilidad analítica $<0,086 \mathrm{pg} / \mathrm{ml}$, una variabilidad intra-ensayo de 2,7$8,1 \%$ y una variabilidad inter-ensayo de $12,6-14,6 \%$.

Se recogieron 6 muestras de saliva por sujeto, entre las 22 p.m. y 3 a.m., en oscuridad a partir de las 24 horas. La última muestra se obtuvo tras exposición directa a una luz blanca brillante de 600 lux durante 1 hora. Las muestras fueron congeladas a $20^{\circ} \mathrm{C}$. La melatonina es estable en saliva a $-20^{\circ} \mathrm{C}$ durante 1 mes (10). Las determinaciones de melatonina se realizaron por duplicado en cada una de las muestras y las muestras del mismo modelo fueron medidas en el mismo ensayo. El test de supresión nocturna de melatonina (TSNM) inducido por exposición a la luz se considera positivo cuando se obtiene un descenso en la concentración de melatonina del 30\% respecto al nivel anterior (11).

\section{Análisis estadístico de los datos}

Los datos se expresaron en valores de medias y desviación estándar (DE). Las diferencias entre
Tabla I. Datos demográficos

\begin{tabular}{|c|c|c|c|}
\hline & $\begin{array}{c}\text { Grupo A } \\
(\mathrm{n}=5)\end{array}$ & $\begin{array}{c}\text { Grupo B } \\
(\mathrm{n}=10)\end{array}$ & $\begin{array}{c}\text { Grupo C } \\
(\mathrm{n}=5)\end{array}$ \\
\hline \multicolumn{4}{|l|}{ EDAD (años) } \\
\hline Media DE & 60,20 DE 3,94 & 61,30 DE 5,47 & 57,20 DE 6,83 \\
\hline Rango & $58-63$ & $51-68$ & $48-65$ \\
\hline \multicolumn{4}{|l|}{ GÉNERO } \\
\hline Hombre & $2(40 \%)$ & $6(60 \%)$ & $5(100 \%)$ \\
\hline Mujer & $3(60 \%)$ & $4(40 \%)$ & $0(0 \%)$ \\
\hline \multicolumn{4}{|l|}{ RAZA } \\
\hline Caucásica & $5(100 \%)$ & $9(90 \%)$ & $5(100 \%)$ \\
\hline Negra & - & $1(10 \%)$ & - \\
\hline
\end{tabular}

DE: desviación estándar.

sujetos controles y pacientes fueron evaluadas estadísticamente con el test t Student. El estudio estadístico se efectuó mediante el programa SPSS 14.0 para Windows (SPSS Inc, Chicago, IL, USA).

\section{RESULTADOS}

Los datos demográficos de los 20 sujetos incluidos en el estudio vienen resumidos en la tabla I. No se encontraron diferencias estadísticamente significativas respecto a la edad entre los 3 grupos de sujetos $(\mathrm{p}=0,5)$.

Los datos obtenidos de la exploración oftalmológica de los sujetos del Grupo A $(n=5)$ fueron normales en todos los casos. El estudio biomicroscópico, medida de la PIO $(14,56 \mathrm{DE} 1,76 \mathrm{~mm} \mathrm{Hg})$ y examen de fondo de ojo fueron normales.

En la tabla II se describen los datos de la evaluación clínica de cada uno de los pacientes del Grupo B

Tabla II. Grupo B. Evaluación clínica

\begin{tabular}{|c|c|c|c|c|c|c|}
\hline Pacientes & Diagnóstico & MAVC (LogMAR) & HVF/DM (dB) & OCT $(\mu \mathrm{m})$ Avg Thick & $m f \mathrm{ERG}$ & TSNM \\
\hline N. ${ }^{0} 1$ & GPAA & 0,16 & $-19,55$ & 47 & Normal & Negativo \\
\hline N. $^{\circ} 2$ & GPAA & 0,25 & $-19,36$ & 44,79 & Normal & Negativo \\
\hline N. $^{\circ} 3$ & GPAA & 0,13 & $-13,75$ & 54,07 & Normal & Negativo \\
\hline N..$^{\circ} 4$ & NOIA & 0,85 & $-22,86$ & 42,81 & Normal & Positivo \\
\hline N. ${ }^{\circ} 5$ & Kjer & 1,00 & $-23,98$ & 59,63 & Normal & Positivo \\
\hline N. ${ }^{\circ} 6$ & GPAA & 0,16 & $-17,81$ & 53,45 & Normal & Negativo \\
\hline N. ${ }^{\circ} 7$ & NOIA & 0,25 & $-20,95$ & 46,75 & Normal & Positivo \\
\hline N. ${ }^{\circ} 8$ & NOIA & 0,64 & $-16,57$ & 55,25 & Normal & Positivo \\
\hline N. ${ }^{\circ} 9$ & Kjer & 0,87 & $-23,84$ & 49,37 & Normal & Positivo \\
\hline N. ${ }^{\circ} 10$ & GPAA & 0,30 & $-26,87$ & 45,40 & Normal & Negativo \\
\hline
\end{tabular}

GPAA: glaucoma primario de ángulo abierto; NOIA: neuropatía óptica isquémica anterior; MAVC: mejor agudeza visual corregida; HVF: Campo visual Humphrey; DM: desviación media; OCT: tomografía de coherencia óptica; Avg ThicK: average thickness; $m f$ ERG: electrorretinograma multifocal. TSNM: test de supresión nocturna de melatonina. 
$(n=10)$. El examen neuro-oftalmológico reveló varias causas de neuropatía óptica: cinco pacientes presentaban glaucoma primario de ángulo abierto (GPAA), tres pacientes neuropatía óptica isquémica anterior (NOIA) y dos atrofia óptica hereditaria tipo Kjer.

La tabla III muestra los valores promedios de las variables analizadas en la exploración oftalmológica de los sujetos del Grupo A y B. Las diferencias entre estos dos grupos fueron estadísticamente significativas en lo que respecta a MAVC LogMAR, DM en HVF y Avg Thickness en el examen OCT $(\mathrm{P}<0,001)$. Las pruebas de $m f E R G$ reflejaron, tanto en el registro tridimensional como en el mapa de ondas, una función normal de los fotorreceptores (fig. 1), en todos los sujetos del Grupo A y B.

Los sujetos del Grupo $\mathrm{C}(\mathrm{n}=5)$ no presentaban PL consciente en $\mathrm{AO}$ y su forma de manejarse y com- portarse era la de una persona ciega. Los reflejos pupilares a la luz estaban abolidos y el examen de fondo de ojo reveló una atrofia óptica en $\mathrm{AO}$, a excepción del Paciente 1 en el cual no se pudieron realizar estas exploraciones por presentar una phthisis bulbi bilateral (tabla IV). Los datos obtenidos de las exploraciones oftalmológicas de estos sujetos no se han incluido en el análisis estadístico. Su colaboración como controles negativos ha quedado restringida a las determinaciones de melatonina en saliva.

\section{Test de supresión nocturna de melatonina (TSNM)}

En el Grupo A, el TSNM fue positivo en el 100\% de los casos. En el Grupo B, cinco de los pacientes

Tabla III. Exploración oftalmológica. Análisis estadístico de datos (Grupos A y B)

\begin{tabular}{|c|c|c|c|c|}
\hline & $\begin{array}{c}\text { MAVC LogMAR (minutos arco) } \\
\text { Media DE }\end{array}$ & $\begin{array}{l}\text { HVF/DM (dB) } \\
\text { Media DE }\end{array}$ & $\begin{array}{c}\text { OCT }(\mu \mathrm{m}) \text { Avg Thich } \\
\text { Media DE }\end{array}$ & $m f \mathrm{ERG}$ \\
\hline \multicolumn{5}{|l|}{ Grupo A $(n=5)$} \\
\hline Sujetos controles & 0,09 DE 0,51 & $0,51 \mathrm{DE} 0,51$ & 113,29 DE 3,94 & Normal \\
\hline \multicolumn{5}{|l|}{ Grupo $B(n=10)$} \\
\hline Sujetos experimentales & 0,46 DE 0,34 & $-20,55$ DE 3,94 & $49,85 \mathrm{DE} 5,46$ & Normal \\
\hline $\mathrm{P}$ & $<0,001$ & $<0,001$ & $<0,001$ & \\
\hline
\end{tabular}

MAVC: mejor agudeza visual corregida; HVF: Campo visual Humphrey; DM: desviación media; OCT: tomografía de coherencia óptica; Avg ThicK: average thickness; mfERG: electrorretinograma multifocal.

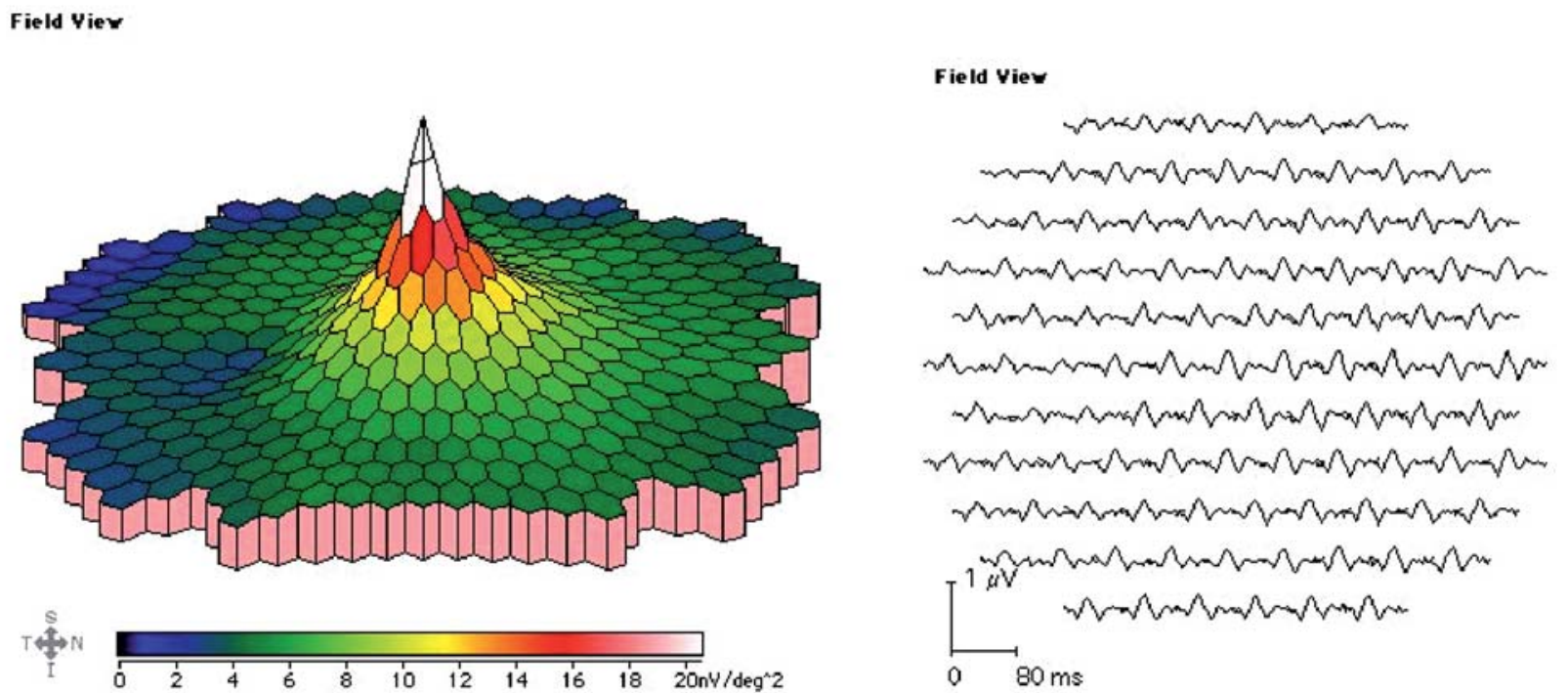

Fig. 1: mfERG. La representación tridimensional y el mapa de ondas reflejan una función normal de la retina externa. 
Tabla IV. Grupo C. Evaluación clínica

\begin{tabular}{|c|c|c|c|c|}
\hline Sujetos & Diagnóstico & AV & Reflejos pupilares & Fondo de ojo \\
\hline N. ${ }^{o} 1$ & Phthisis bulbi AO & No PL & No explorables & No explorable \\
\hline N. ${ }^{\circ} 2$ & GPAA AO & No PL & Abolidos & Atrofia óptica AO \\
\hline N. 3 & Glaucoma congénito & No PL & Abolidos & Atrofia óptica AO \\
\hline N. ${ }^{\circ} 4$ & Retinopatía de la prematuridad & No PL & Abolidos & No explorable \\
\hline N. ${ }^{\circ} 5$ & Neuropatía óptica de Leber & No PL & Abolidos & Atrofia óptica $\mathrm{AO}$ \\
\hline
\end{tabular}

AV: agudeza visual; PL: percepción luminosa; AO: ambos ojos.

presentaron un TSNM negativo (50\% casos) y otros cinco pacientes lo presentaron positivo (50\% casos) (tabla II). En el Grupo C el TSNM fue negativo en el $100 \%$ casos.

\section{DISCUSIÓN}

En nuestra serie, el 100\% de los sujetos ciegos del Grupo C, en ausencia de PL consciente y con reflejos pupilares abolidos, presentaron un TSNM negativo. Sin embargo, los resultados del TSNM en los pacientes del Grupo B, afectos de neuropatías ópticas severas en $\mathrm{AO}$ y con grados variables de agudeza visual, fueron más heterogéneos: el TNSM fue negativo sólo en el $50 \%$ de los casos.

El TSNM por exposición a la luz podría ser de gran utilidad para determinar la integridad funcional del TRH, que a través del NSQ conecta la retina con la glándula pineal (12). En este sentido, se ha demostrado que algunas personas ciegas, en ausencia de percepción consciente de luz, exhiben supresión nocturna de melatonina (11) y ritmos circadianos de secreción de melatonina sincronizados a 24 horas $(13,14)$, lo que indicaría que la luz actúa a nivel del NSQ. Datos similares obtenidos en trabajos de experimentación animal con ratas apoyan estos resultados (15).

El hecho de que personas ciegas mantengan sus respuestas no visuales a la luz $(11,13,14)$, apoyaría la hipótesis de que en el ojo de los mamíferos existan al menos dos sistemas anatómica y funcionalmente diferentes que detectan luz: 1) por una parte, el sistema visual clásico que forma imágenes y que involucra a los conos y bastones, 2) por otra parte, un sistema fotorreceptivo que detecta luz, pero que no forma imágenes, y que sincroniza los ritmos circadianos. El TRH que se origina en una subpoblación de células ganglionares de la retina sensible a la luz, las ipCGR, proyecta NSQ y forma parte de este sistema (16).
Un gran avance en el conocimiento de cómo la luz estimula funciones no visuales, vino dado por el descubrimiento de una nueva opsina, la melanopsina, en el ojo de los mamíferos (17). Sorprendentemente, la melanopsina no se localiza en los fotorreceptores, donde se hallan la rodopsina de los bastones y las opsinas de los conos, sino en las ipCGR (18).

Como el efecto de la luz sobre la supresión de melatonina es dosis-dependiente (19), cabría pensar que la severidad de la pérdida visual y el tipo de enfermedad podrían condicionar este efecto. En estudios clínicos realizados en pacientes con disfunciones del sistema de conos o con retinosis pigmentaria, se ha demostrado que la pérdida visual tiene escasa repercusión sobre el sistema circadiano $(20,13)$. En nuestra serie, todos los pacientes estudiados presentaban neuropatías ópticas severas en ambos ojos con función normal de su retina externa demostrada por $m f E R G$, pero a pesar de la pérdida tan intensa de células ganglionares de la retina, el $50 \%$ de estos pacientes exhiben un TSNM positivo, quizás debido a una mayor resistencia de las ipCGR a la lesión en estas enfermedades (21). También, hemos observado que el $100 \%$ de pacientes con GPAA mostraban un TSNM negativo, lo que nos sugiere que en nuestra serie las ipCGR son más sensibles al daño glaucomatoso.

A partir de estos resultados preliminares podemos concluir que el 50\% de los pacientes con neuropatías ópticas severas exhiben alteraciones en las respuestas no visuales a la luz, en términos de supresión de la secreción nocturna de melatonina, lo que implicaría una disfunción de la ipCGR a pesar de la normalidad funcional de los fotorreceptores. Estudios adicionales (p.ej. los potenciales evocados multifocales) nos podrían aportar información del grado de funcionalidad de las células ganglionares de la retina en estos pacientes con objeto de predecir su posible asociación con trastornos de los ritmos circadianos. 


\section{BIBLIOGRAFÍA}

1. Reiter RJ. Pineal melatonin: Cell biology of its synthesis and of its physiological interactions. Endocrine Reviews 1991; 12: 151-180.

2. Freedman MS, Lucas RJ, Soni B, Von Schantz, M, Muñoz $M$, David-Gray Z, et al. Regulation of mammalian circadian behaviour by non-rod, non-cone, ocular photoreceptors. Science 1999; 284: 502-504.

3. Panda S, Nayak SK, Campo B, Walter JR, Hogenesh JB, Jegla T. Illumination of the melanopsin signaling pathway. Science 2005; 307: 600-604.

4. Moore RY, Speh JC, Card JP. The retino-hypothalamic tract originates from a distinct subset of retinal ganglion cells. J Comp Neurol 1995; 352: 351-366.

5. Larsen PJ, Enquist LW, Card JP. Characterization of the multisynaptic neuronal control of the rat pineal gland using viral transneuronal tracing. Eur J Neurosci 1998; 10: $128-145$.

6. Lewy AJ, Wehr TA, Goodwin FK, Newsome DA, Markey SP. Light supresses melatonin secretion in humans. Science 1980; 210: 1267-1269.

7. Webb SM, Puig-Domingo M. Role of melatonin in health and disease. Clin Endocrinol 1995; 42: 221-234.

8. Arendt J, Bojkowski C, Franey C, Wright J, Marks V. Immunoassay of 6-hydroxymelatonin sulfate in human plasma and urine: abolition of the urinary 24-hour rhythm with atenolol. J Clin Endocrinol Metab 1985; 60: 1166-1173.

9. Nowak R, McMillen IC, Redman J, Short RV. The correlation between serum and salivary melatonin concentrations and urinary 6-hydroxymelatonin sulphate excretion rates: two non-invasive techniques for monitoring human circadian rhythmicity. Clin Endocrinol 1987; 27: 445-452.

10. Simonin G, Bru L, Lelievre E, Jeanniot JP, Bromet N, Walther B, et al. Determination of melatonin in biological fluids in the presence of the melatonin agonist S 20098: comparison of immunological techniques and GC-MS methods. J Pharm Biomed Anal 1999; 21: 591-601.
11. Czeisler CA, Shanahan TL, Klerman EB, Martens H, Brotman DJ, Emens JS, et al. Suppression of melatonin secretion in some blind patients by exposure to bright light. $N$ Engl J Med 1995; 332: 6-11.

12. Reppert SM. Circadian rhythms: basic aspects and pediatric implications. In: Styne DM, Brook CGD. Current concepts in pediatric endocrinology. New York: Elsevier; 1987; 91-125.

13. Lockley SW, Skene DJ, Arendt J, Tabandeh H, Bird AC, Defrance R. Relationship between melatonin rhythms and visual loss in the blind. J Clin Endocrinol Metab. 1997; 82: $3763-3770$

14. Klerman EB, Shanahan TL, Brotman DJ, Rimmer DW, Emens JS, Rizzo JF 3rd, Czeisler CA. Photic resetting of the human circadian pacemaker in the absence of conscious vision. J Biol Rhythms 2002; 17: 548-555.

15. Provencio I, Wong $S$, Lederman AB, Argamaso SM, Foster $R G$. Visual and circadian responses to light in aged retinally degenerate mice. Vision Res 1994; 34: 1799-1806.

16. Berson DM, Dunn FA, Takao M. Phototransduction by retinal ganglion cells that set the circadian clock. Science 2002; 295: 1070-1073.

17. Provencio I, Rodriguez IR, Jiang G, Hayes WP, Moreira $E F$, Rollag MD. A novel human opsin in the inner retina. $J$ Neurosci 2000; 20: 600-605.

18. Gooley JJ, Lu J, Chou TC, Scammell TE, Saper CB. Melanopsin in cells of origin of the retinohypothalamic tract. Nat Neurosci 2001; 4: 1165.

19. Lewy AJ, Wehr TA, Goodwin FK. Light suppresses melatonin secretion in humans. Science. 1980; 210: $1267-$ 1269.

20. Ruberg FL, Skene DJ, Hanifin JP, Rollaq MD, English J. Melatonin regulation in humans with color vision deficiencies. J Clin Endocrol Metab 1996; 81: 2980-2985.

21. Robinson GA, Madison RD. Axotomized mouse retinal ganglion cells containing melanopsin show enhanced survival, but not enhanced regrowth into a peripheral nerve graft. Vision Res 2004; 44: 2667-2674. 
